\title{
Evidence-based recommendations for the organisation of long-term follow-up care for childhood and adolescent cancer survivors: A report from the PanCareSurFup Guidelines Working Group
}

Gisela Michel, $\mathrm{PhD}^{\mathrm{a}^{* \dagger}}$ Renée L Mulder, PhD ${ }^{\mathrm{b}^{*}}$ Helena JH van der Pal, MD PhD ${ }^{\mathrm{b}}$ Roderick Skinner, FRCPCH ${ }^{\mathrm{c}, \mathrm{d}}$ Edit Bárdi MD, PhD ${ }^{\mathrm{e}, \mathrm{f}}$ Morven C Brown, D Health Psych ${ }^{\mathrm{g}}$ Janine Vetsch, PhD $^{\mathrm{a}, \mathrm{h}, \mathrm{i}}$ Eva Frey, $\mathrm{MD}^{\mathrm{e}}$ Rachael Windsor, MD ${ }^{\mathrm{j}}$ Leontien CM Kremer, MD PhD ${ }^{\mathrm{b}, \mathrm{k}^{* *}}$ Gill Levitt, $\mathrm{MD}^{1 * *}$

* Joint first authorship

** Joint-last authorship

$\dagger$ corresponding author

a Department of Health Sciences and Health Policy, University of Lucerne, Lucerne, Switzerland, gisela.michel@unilu.ch

b Princess Máxima Center for Pediatric Oncology, Utrecht, the Netherlands, R.L.Mulder@prinsesmaximacentrum.nl,H.J.H.vanderPal@prinsesmaximacentrum.nl, L.C.M.Kremer@prinsesmaximacentrum.nl

${ }^{c}$ Northern Institute for Cancer Research, Newcastle University, Newcastle upon Tyne, United Kingdom, roderick.skinner@newcastle.ac.uk

d Department of Paediatric and Adolescent Haematology/Oncology and Children's Haemopoietic Stem Cell Transplant Unita, Great North Children's Hospital, Newcastle upon Tyne Hospitals NHS Foundation Trust, Newcastle upon Tyne, United Kingdom

e St Anna Children's Hospital, Wien, Austria, eva.frey@a1.net

${ }^{\mathrm{f}}$ Kepler Universitätsklinikum, Linz, Austria, editbardi@hotmail.com

g Institute of Health \& Society, Newcastle University, Newcastle upon Tyne, United Kingdom, morven.brown@newcastle.ac.uk

h School of Women's and Children's Health, UNSW Sydney, Australia,j.vetsch@unsw.edu.au

i Behavioural Sciences Unit, Kids Cancer Centre, Sydney Children’s Hospital, High Street, Randwick, NSW, Australia, 2031

j Children and Young People's Cancer Service, University College London Hospitals NHS Foundation Trust, London, United Kingdom, rachael.windsor@nhs.net

k Emma Children's Hospital, Amsterdam UMC, University of Amsterdam, Paediatric Oncology, Amsterdam, the Netherlands

${ }^{1}$ Department of Paediatric Oncology, Great Ormond Street Hospital for Children, NHS Foundation Trust, London, United Kingdom, gill@hebber.co.uk

Corresponding author:

Gisela Michel, Department of Health Sciences and Health Policy, University of Lucerne

Frohburgstrasse 3, 6002 Luzern, Switzerland

Ph: +41 41 2295955, Fax: +41 41 2295635, E-Mail: gisela.michel@unilu.ch

\section{Acknowledgement}

This work was supported by the European Union's Seventh Framework Programme for research, technological development and demonstration under grant agreement no 257505 (PanCareSurFup).

Citation: Michel G, Mulder RL, van der Pal HJH, Skinner R, Bárdi E, Brown MC, et al. Evidence-based recommendations for the organization of long-term follow-up care for childhood and adolescent cancer survivors: a report from the PanCareSurFup Guidelines Working Group. Journal of Cancer Survivorship. 2019. https://doi.org/10.1007/s11764-019-00795-5 


\title{
Evidence-based recommendations for the organisation of long-term follow-up care for childhood and adolescent cancer survivors: A report from the PanCareSurFup Guidelines Working Group
}

Gisela Michel, $\mathrm{PhD}^{\mathrm{a}^{* \dagger}}$ Renée L Mulder, $\mathrm{PhD}^{\mathrm{b}^{*}}$ Helena JH van der Pal, MD PhD ${ }^{\mathrm{b}}$ Roderick Skinner, FRCPCH ${ }^{\mathrm{c}, \mathrm{d}}$ Edit Bárdi MD, PhD ${ }^{\mathrm{e}, \mathrm{f}}$ Morven C Brown, D Health Psych ${ }^{\mathrm{g}}$ Janine Vetsch, PhD ${ }^{\mathrm{a}, \mathrm{h}, \mathrm{i}}$ Eva Frey, $\mathrm{MD}^{\mathrm{e}}$ Rachael Windsor, MD ${ }^{\mathrm{j}}$ Leontien CM Kremer, MD PhD ${ }^{\mathrm{b}, \mathrm{k}^{* *}}$ Gill Levitt, $\mathrm{MD}^{1^{* *}}$

\begin{abstract}
Purpose: To facilitate the implementation of long-term follow-up (LTFU) care and improve equality of care for childhood, adolescent, and young adult (CAYA) cancer survivors, the PanCareSurFup Guidelines Working Group developed evidence-based recommendations for the organisation of LTFU.

Methods: We established an international multidisciplinary guideline panel. A systematic review of the literature published from 1999-2017 was completed to answer six clinical questions. The guideline panel reviewed the identified studies, developed evidence summaries, appraised the quality of the body of evidence and formulated recommendations based on the evidence, expert opinions, and the need to maintain flexibility of application across different healthcare systems.

Results: We provide strong recommendations based on low level evidence and expert opinions, regarding organization of LTFU care, personnel involved in LTFU care, components of LTFU care and start of LTFU care. We recommend that risk adapted LTFU care provided under the guidance of a cancer survivorship expert service or cancer centre should be available and accessible for all CAYA cancer survivors throughout their lifespan.

Conclusion: Despite the weak levels of evidence, successful and effective implementation of these recommendations should improve LTFU, thereby leading to better access to appropriate healthcare services and an improvement in health outcomes for CAYA cancer survivors.

Implications for cancer survivors: To improve health outcomes and quality of survivorship of current and future survivors, continued age-adapted education of survivors about the cancer, its treatment, risk for late-effects, importance of health behaviours, and necessity of LTFU is important along the cancer and survivorship trajectory.
\end{abstract}

\section{Keywords (MESH):}

Evidence-based guideline; recommendations; follow-up care; oncology; survivor; child; adolescent

\section{Introduction}

Long-term follow-up (LTFU) care is important for childhood, adolescent, and young adult (CAYA) cancer survivors due to their high risk of late-effects [1-5]. Prevention, early detection, and management of late-effects are recommended to improve the quality of survivorship. Additionally, follow-up provides an opportunity to offer age-appropriate information about disease, treatment, and a healthy lifestyle, as well as practical advice about insurance, education or work, and psychosocial support [6-8]. With increasing age, survivors need to increase the personal responsibility for the management of their own health [9-11]. This is particularly relevant for survivors who are transitioned to adult care. Several guidelines recommend risk-stratified LTFU for CAYA cancer survivors [12-15]. However, there is a lack of LTFU care for paediatric and especially adult survivors of CAYA cancer in many countries [16]. Also, there is currently no evidence-based guideline on how to organise follow-up care to deliver the recommended content of LTFU effectively (e.g. surveillance, education). In a recent survey, we found that paediatric oncologists/haematologists and late-effect specialists across Europe agreed that guidelines and recommendations for LTFU are needed [17].

To facilitate the development of evidence-based LTFU services and improve quality of care for CAYA cancer survivors, the PanCareSurFup (PCSF) Guidelines Working Group aimed to develop a pan-European guideline for the organisation of LTFU. 


\section{Methods}

We used evidence-based methods to systematically search, and review the literature, extract the evidence, and formulate recommendations. We followed the methods reported by the International Guideline Harmonization Group (www.IGHG.org)[18, 19]. The working group comprised 11 experts from paediatric and medical oncology, survivorship care, guideline methodology, psychology, and epidemiology (see author list).

\section{Scope of the guideline}

The scope of this guideline is to provide healthcare providers, patients, survivors, and parents/caregivers with recommendations for the provision of LTFU care for CAYA cancer survivors diagnosed with cancer up to 25 years of age and at least 2 years after the completion of treatment.

\section{Systematic literature review}

First, we defined four key issues that should be addressed: organization of LTFU care; personnel involved in LTFU care; components of LTFU care; start of LTFU care. For each key issue we assessed the concordance and discordance among five existing guidelines for CAYA cancer survivors: Scottish Intercollegiate Guidelines Network,[20] Dutch Childhood Oncology Group (DCOG)[13], United Kingdom Children's Cancer and Leukaemia Group (CCLG)[14] , North American Children's Oncology Group (COG)[15], Australian Cancer Survivorship Centre[21](Online Resource 1).

Second, based on the identified discordances we defined six clinical questions covering the formulated key issues about LTFU care for CAYA cancer survivors: 1) What are the opinions of survivors and healthcare providers about LTFU? (specifically, issues such as who should be involved in LTFU? What are the key requirements of LTFU? What are the barriers to LTFU? What are the facilitators of LTFU?); 2) What theoretical models of LTFU care exist?; 3. What models of survivorship care are used in practice?; 4) What is the effectiveness of survivorship care models in practice?; 5) What is the effectiveness of risk stratification?; 6) What is the effectiveness of childhood cancer survivorship care plans?

\section{Search strategy and selection criteria}

To update a previous systematic search developed by the DCOG guideline (January 1999-2009)[13] we conducted a systematic literature search in PubMed in 2014 and updated the search in February 2017 (the updated search resulted in more evidence but no change of previous conclusions and recommendations). The search terms used are listed in Table 1. In addition, we searched the reference lists of the existing guidelines and existing reviews, and contacted survivorship care experts to determine if any additional evidence was available. We included papers if the majority of participants were CAYA cancer survivors (i.e. $\geq 55 \%$ of sample diagnosed before the age of 25 years) and they were published in English. For clinical questions 1-3 we included all types of study designs (cohort studies, reviews, qualitative studies, expert papers). For clinical questions 4-6 we initially aimed to include randomized controlled trials (RCTs) or cohort studies that compared different models of care or with pre- and postmeasurements. However, due to a lack of such studies, we also included other type of study designs.

Title/abstract and then full text of each paper were reviewed by two independent members of the working group for inclusion/exclusion in the development of recommendations, and it was decided for which clinical questions the study should be included. This work was shared amongst all authors. Where disagreement occurred the paper was discussed between the two reviewers, and if disagreement persisted, between the remaining members of the working group. We generated evidence summaries using standardized data-abstraction forms to answer the clinical questions. We graded the quality of the evidence according to evidence-based methods (Online Resource 2)[18].

\section{From evidence to recommendations}

The working group reached consensus on final recommendations based upon the evidence, expert opinions (via a panel of international experts and CAYA cancer survivors at a PanCare meeting in Erice in November 2016 [22]), and the need to maintain flexibility of application across different healthcare systems. Decisions were made through group discussion and consensus, and final recommendations had to be supported unanimously. The strength of 
the recommendations were graded according to published evidence-based methods (Online Resource 2)[18].

\section{Results}

The search in PubMed yielded 2823 abstracts, of which 273 were included for full text search. In total, 84 papers were included in this guideline (Figure 1). The conclusions of evidence for the six questions are presented in Table 2 and in more detail in Online Resource 3 , and the evidence tables for each paper are available in Online Resource 4.

The final recommendations are shown in Table 3. All recommendations were based on a combination of low-quality evidence (level C) and expert opinions because no high level (A/B) evidence was identified. Below we describe the recommendations and their rationale.

\section{Organization of LTFU care}

\section{Evidence}

Different models of survivorship care are described in the literature, of which the cancer centre-delivered care in the paediatric oncology clinic, medical oncology clinic or LTFU clinic are the most common models [16, 23-38]. Two other described models are the primary care physician-led LTFU care [27, 37, 39-42], and shared care between treatment hospital and local hospital or primary care [37, 40]. Risk stratification is seen as an important component of LTFU [43-45] with a variety of models described in CAYA cancer survivors [34, 43, 44, 46-48]. Risk stratification is based on cancer treatment, diagnosis and current health status. There is no evidence that one stratification model is better than the other. Studies have shown that it is possible to both identify [49, 50], and categorize survivors correctly [34, 43 , 44, 46-48]. Frobisher et al showed that survivors classified into a higher risk group according to their cancer diagnosis and treatment were more likely to be diagnosed with subsequent primary neoplasms, as well as fatal and non-fatal non-neoplastic outcomes than the lower risk groups [50].

Survivors at low risk can be adequately followed-up by a primary care physician-led LTFU (community-based care) model [44, 45, $51,52]$. Some evidence suggests that primary care physicians are both willing and able to provide LTFU care for adult survivors of childhood cancer $[27,39,42,53]$, but they sometimes may feel uncomfortable [54-57]. Primary care providers reported several barriers to successful LTFU care: lack of communication between primary care physician and paediatric oncologists $[42,58]$ (which was also mentioned by oncologists $[59,60]$ ), limited access to refer survivors to specialist care (i.e. oncology, mental health, non-cancer care)[61], and their overall workload $[42,58]$. Adequate access to cancer survivor specialists [54, 61], and support services such as social work and psychology [54], the ability to contact specialists for advice by telephone or email [54], and more medical/support staff in the primary care office[54] can facilitate the involvement of primary care providers in LTFU.

Some evidence suggests that for survivors at moderate risk of late-effects LTFU care can be provided by a primary care physician $[44,45$, $51,52]$, or a nurse experienced in management of late effects [43, 45], followed by supported self-management [43-45]. Follow-up at a cancer survivorship expert centre is an option as well [43, 45, 51, 52]. In addition, shared-care between the cancer survivorship expert centre and primary care or paediatric centres has been proposed [43, 44, 52]. Some evidence suggests that LTFU within a shared-care model is feasible [62], and that the vast majority of survivors and primary care physicians were satisfied with the follow-up care [37, 62]. In addition, when primary care and subspecialty survivorship care providers were compared there were no significant differences in survivors' satisfaction and engagement in LTFU [63]. The shared-care model has been successfully implemented, though less frequently than the cancer-centre delivered care model $[42,53]$.

Some evidence suggests that for high risk survivors LTFU can be provided in a cancer centre-delivered care model in the paediatric oncology clinic, medical oncology clinic or LTFU clinic and many clinics around the world have already adopted this model [16, 23-30, 32, 34-36, 38, 64]. However, distance to the clinic for survivors,[16] and the inability to locate adult survivors with increasing time since treatment[16, 23] have been recognised as potential barriers to attend LTFU. Other reported barriers to successful LTFU is lack of support, both institutional [59], or from 
colleagues $[16,25]$, and especially a lack of health insurance coverage for survivors[16, 23, $25,59,60,65-68]$. In addition, lack of dedicated time was reported by primary care physicians $[61,65]$, and oncologists $[16,25,69]$ as an important barrier.

Overarching is the necessity of the provision of multidisciplinary care $[16,24-30,32,34-36,38$, $41,42,53]$. Studies reported that paediatric oncologists preferably provide LTFU care in a multidisciplinary team $[16,70,71]$. Parents of survivors prefer a multidisciplinary team model as well [72].

\section{Recommendations}

Based on expert opinion and low-quality evidence, we recommend that LTFU care should be available and accessible for all CAYA cancer survivors throughout their lifespan. Due to their diverse needs, CAYA survivorship care should be provided under the guidance of a cancer survivorship expert service or cancer centre, preferably in a multidisciplinary setting. The adult CAYA cancer survivor and healthcare provider should make the decision for the optimal model of LTFU care together based on previous cancer treatment, diagnosis and health status, survivor preferences and taking in to account the available healthcare system. The model of care for children will depend on local policies.

Regular review of the level of care and flexibility herein is important, especially the possibility for quick referral back into the cancer survivorship care system. Moreover, we recommend that (national and local) healthcare providers (systems) and insurers have commitment to provide LTFU care for survivors and allocate sufficient time (strong recommendations).

\section{Personnel involved in LTFU care}

\section{Evidence}

Some evidence suggests that despite the various models recommended for survivors at different risks for late-effects, experts and survivors agree that survivors should make their own choice for their LTFU provider [22, 67]. A successful model of LTFU needs the flexibility to adapt to the individual survivor. This is especially important because many survivors prefer hospital-based LTFU, while others would rather attend LTFU at their primary care provider [72-75]. Also in contrast to expectations, many childhood cancer survivors who attend a paediatric clinic are significantly more [76], or equally[77] satisfied with LTFU care than survivors attending an adult clinic. This is important because in many clinics LTFU for adult CAYA cancer survivors is still provided at the paediatric hospital [64], although studies suggest that paediatric oncologists do not feel very comfortable caring for older adults $[70,71]$, and some healthcare systems do not allow adult survivors to be seen in paediatric settings. To empower survivors in making their choice of provider, the evidence and our expert panel in Erice[22] agreed on the importance of a key worker or coordinator of care [41]. Other important LTFU staff that should be available at a cancer survivorship expert centre as reported in the literature are a lead doctor specialised in late-effects, and a nurse practitioner [78]. Lack of staff to provide LTFU has been indicated to be a major barrier $[16,59]$.

\section{Recommendations}

Based on expert opinion and low-quality evidence, we recommend that each CAYA cancer survivor should make their own informed choice for a healthcare provider after discussion with the survivorship team. The cancer survivorship expert centre that will organize LTFU care should include a key worker/coordinator, lead doctor specialised in late effects, nurse practitioner, and a multidisciplinary expert team of specialists, like paediatric oncologist/haematologist, (neuro-) psychologist, cardiologist, endocrinologist, medical oncologist, haematologist, rehabilitation physician, occupational worker, radiotherapist, and social worker. In addition, it is important that there is the possibility of consulting other specialist, like pulmonologist, nephrologist, neurologist, neurosurgeon, ear nose and throat specialist, ophthalmologist, gynaecologist, dermatologist, insurance worker, urologists, and general internal medicine. It is important to keep in mind that all aspects of LTFU might need to be adapted to the healthcare system in place (strong recommendations).

\section{Components of LTFU care}

\section{Evidence}

Survivors[66, 67, 73-75, 79-83] and their parents[72, 84] reported a wide-range of needs for LTFU. One of the main core components of 
LTFU care is the provision of information, counselling and surveillance for late effects, and preventive care based on evidence-based guidelines. Primary care physicians $[54,55,58$, $69,85]$ and oncologists $[69,70]$ report the need for guidelines, and a lack thereof is seen as an important barrier to successful LTFU by primary care physicians $[54,55,61,65]$, oncologists [70]. and survivors [66]. The ideal format may be one that can be instantly accessed during the follow-up appointment [85].

Another important core component is the coordination of care. There is potential confusion between the different roles of survivorship programs, oncologists, and primary care providers [60]. Experts have highlighted the importance of well-coordinated care (particularly in shared care models) and proposed the inclusion of a key worker [43, 44, 86].

Education of professionals involved is essential. Some evidence suggests that a lack of knowledge and experience is seen as a barrier to providing LTFU care by primary care physicians [42, 58, 61], oncologists [69], and policy experts [87], and the need for continued education is widely accepted $[30,34,42,43,54$, 69]. Online tools are seen as a suitable possibility to reach many professionals. [54, 85] Education of survivors, families, and carers to increase awareness and knowledge about cancer and treatment-related risks of late-effects, and the importance of LTFU care is necessary as well. This has been highlighted by experts[ $[43$, $86]$ but is also shown in practice $[16,25,28,30$, $31,33,39,42]$. Studies showed that the lack of knowledge is not only seen as a barrier to successful LTFU by oncologists [16, 23, 25, 59], but also by survivors[10, 79, 88] and parents themselves[84, 88]. In addition, some evidence suggests that a survivorship clinic, including survivor education, may improve survivors' knowledge of their treatment history and risk of late-effects [33], may increase awareness of personal health risk [31], and may decrease emergency department visits [89].

Another core component of LTFU care is the coordination of scientific research to improve evidence-based care and to audit its effectiveness [42]. One US study showed that three quarters of 179 responding survivorship programs had a research associate available [25],

An important tool for successful LTFU is the individualised survivorship care plan (SCP) including a treatment summary $[16,25,28,30$, $33,35,39,41-44,86]$. A SCP has been reported as being of great importance by survivors $[10$, $37,53,62,73,90-92]$, oncologists [70], and also primary care providers [55, 58, 61, 65, 93]. Some evidence suggests that primary care providers and survivors are satisfied with a SCP and that it may increase knowledge of late effects and awareness of the benefits of LTFU $[39,91,93]$. In addition, a SCP may improve communication with survivors about lateeffects and the sharing of more comprehensive information $[94,95]$. It may support healthcare providers' adherence to guidelines on lateeffects screening and survivors' compliance with recommended medical surveillance [94, 95]. However training of providers is essential [57].

Effective self-management is an important component of survivorship care. To achieve this aim, survivor and parent education is vital, especially when survivors reach adulthood and take over responsibility for their health. Some evidence suggests that survivors reported low confidence in managing their survivorship care [96], including problems organising appointments [68], or finding the right place to go [80].

Another aspect of high importance to successful LTFU of adult CAYA cancer survivors is their transition from paediatric to adult care [43, 44, 86]. Oncologist and healthcare policy experts agree that the lack of a transition programme is a major barrier [16, 87], despite evidence that survivors are willing to transition to adult survivorship care [37, 97]. Several institutions have already successfully implemented planned transition in their programmes $[25,26,30,35$, $36,42,98]$.

\section{Recommendations}

We recommend that LTFU care for CAYA cancer survivors include 1) surveillance and preventive strategies based on published evidence-based guidelines; 2) coordination of care (particularly in shared care models); 3) education for professionals; 4) education of survivors, families, and carers; and 5) coordination of scientific research. Based on 
expert opinion and low-quality evidence, we recommend that an individualized SCP is provided to every CAYA cancer survivor at the end of treatment, including a treatment summary with a care plan. In addition, the cancer survivorship expert centre should provide survivor and parent education to support effective self-management and a plan for transition of care from active treatment to LTFU, from survivorship expert centre to primary care (for low risk survivors) and from paediatric to adult health service (strong recommendations).

\section{Start of LTFU care}

Recommendations

Although there is currently no evidence available, our expert panel agreed that LTFU is recommended to start no later than 5 years after treatment or 5 years from diagnosis, depending on the healthcare system (strong recommendation).

\section{Discussion}

This systematic review of available evidence and expert panel discussions confirmed the importance of LTFU care for CAYA cancer survivors. We have made comprehensive recommendations for delivering effective LTFU care, relevant to healthcare providers, survivors and their families, and researchers.

Our recommendations describe the minimal requirements for LTFU. The exact organisation of care will depend on the national healthcare system, regional characteristics, features of the hospital and finally preferences and needs of survivors and healthcare providers. In the UK, for example, the National Cancer Survivorship Initiative (NCSI) has been developed by experts and stakeholders, and resulted in similar recommendations as we concluded from our evidence [99]. A one-stop survivorship clinic, including access to a multidisciplinary team, when needed, within one appointment, would provide a suitable system decreasing organisational barriers for survivors. Another important aspect is easy accessibility for all survivors, especially those who might not have attended LTFU for a long time and whom wish to return to follow-up care.

Despite the overall lack of clinical trials addressing the impact of LTFU on survivors' medical and psychosocial outcomes, we showed that there is some evidence for a positive effect: survivors attending LTFU demonstrated higher knowledge about their diagnosis and treatment, more accurate perception of their risk for late-effects $[31,33]$, were engaged in regular surveillance, $[29,94]$ and had fewer visits to the emergency department [89]. In addition, a recent review also showed that survivors attending LTFU care had more late-effects detected [100]. This demonstrates the importance of LTFU.

We also provide evidence for the usefulness of an individualized SCP. However, clear evidence for the effectiveness of an SCP to improve LTFU and survivor's health in the long term is still lacking [101]. Furthermore, followup recommendations will change with new and updated guidelines and survivors' follow-up care will therefore need to be reviewed regularly and adapted if necessary.

More than 10 years after the report "From Cancer Patient to Cancer Survivor: Lost in Transition" by the Institute of Medicine [102] there has been a large amount of research and publications addressing LTFU of CAYA cancer survivors. However, our literature review still showed some important gaps in knowledge. While opinions of survivors, parents, providers, and experts have been assessed frequently and LTFU implemented in many places, there is still a lack of studies on effectiveness. The models themselves, the provision of care including risk stratification, but also specific interventions such as an SCP need to be evaluated for their effectiveness to detect late effects, improve survivors' quality of life and knowledge acquisition as well as their cost-effectiveness. An update of our guideline in 5 years will hopefully be able to include stronger evidence of effectiveness.

To improve health outcomes and quality of survivorship of current and future survivors, continued age-adapted education of survivors about the cancer, its treatment, risk for lateeffects, importance of health behaviours, and necessity of LTFU is important along the cancer and survivorship trajectory. Well-organised LTFU will help to reach this goal. 


\section{Compliance with Ethical Standards:}

\section{Funding}

This work was supported by the European Union's Seventh Framework Programme for research, technological development and demonstration under grant agreement no 257505 (PanCareSurFup).

\section{Role of the funding source}

The funding sources had no role in any parts of the study.

\section{Conflict of Interest:}

The authors declare that they have no conflict of interest.

\section{Ethical approval:}

This article does not contain any studies with human participants performed by any of the authors.

\section{References}

1. Hudson, M.M., et al., Age-Dependent Changes in Health Status in the Childhood Cancer Survivor Cohort. J Clin Oncol, 2015. 33(5): p. 479-491.

2. Geenen, M.M., et al., Medical assessment of adverse health outcomes in long-term survivors of childhood cancer. JAMA, 2007. 297(24): p. 2705-15.

3. Hudson, M.M., et al., Clinical ascertainment of health outcomes among adults treated for childhood cancer. JAMA, 2013. 309(22): p. 2371-81.

4. Oeffinger, K.C., et al., Chronic health conditions in adult survivors of childhood cancer. N Engl J Med, 2006. 355(15): p. 1572-82.

5. Bitsko, M.J., et al., Psychosocial Late Effects in Pediatric Cancer Survivors: A Report From the Children's Oncology Group. Pediatr Blood Cancer, 2016. 63(2): p. 337-343.

6. von der Weid, N. and H.P. Wagner, Organisation of follow-up in paediatric oncology. Eur J Cancer, 2003. 39(8): p. 1150-1154.

7. Gianinazzi, M.E., et al., Information provision and information needs in adult survivors of childhood cancer. Pediatr Blood Cancer, 2014. 61(2): p. 312318.

8. Vetsch, J., et al., Information needs in parents of longterm childhood cancer survivors. Pediatr Blood Cancer, 2015. 62(5): p. 859-866.

9. Stinson, J.N., et al., Disease self-management needs of adolescents with cancer: perspectives of adolescents with cancer and their parents and healthcare providers. J Cancer Surviv, 2012. 6(3): p. 278-86.

10. Zebrack, B.J., et al., Health care for childhood cancer survivors: insights and perspectives from a Delphi panel of young adult survivors of childhood cancer. Cancer, 2004. 100(4): p. 843-50.

11. Syed, I.A., et al., Examining factors associated with self-management skills in teenage survivors of cancer. J Cancer Surviv, 2016. 10(4): p. 686-91.
12. Scottish Intercollegiate Guidelines Network (SIGN), Long term follow-up of survivors of childhood cancer. A national clinical guideline, No. 76. 2004, Edinburgh: Scottish Intercollegiate Guidelines Network.

13. Stichting Kinderoncologie Nederland (SKION). Richtlijn follow-up na kinderkanker meer dan 5 jaar na diagnose. 20105 October 2011 [cited 201628 July]; Available from: https://www.skion.nl/voorpatienten-en-ouders/late-effecten/533/richtlijnfollow-up-na-kinderkanker/.

14. Skinner, R., H. Wallace, and G. Levitt, Therapy based long term follow up: practice statement. 2005, Leicester: United Kingdom Children's Cancer Study Group (Late Effects Group).

15. Children's Oncology Group. Long-Term Follow-Up Guidelines for Survivors of Childhood, Adolescent, and Young Adult Cancers V4.0. 2013 [cited 201628 July]; Available from: http://www.survivorshipguidelines.org/.

16. Essig, S., et al., Follow-up programs for childhood cancer survivors in Europe: a questionnaire survey. PLoS One, 2012. 7(12): p. e53201.

17. Brown, M.C., et al., The views of European clinicians on guidelines for long-term follow-up of childhood cancer survivors. Pediatr Blood Cancer, 2015. 62(2): p. $322-328$

18. Kremer, L.C., et al., A worldwide collaboration to harmonize guidelines for the long-term follow-up of childhood and young adult cancer survivors: a report from the International Late Effects of Childhood Cancer Guideline Harmonization Group. Pediatr Blood Cancer, 2013. 60(4): p. 543-9.

19. Mulder, R.L., et al., Handbook for guideline development; collaboration between International Guideline Harmonization Group, PanCareSurFup and Cochrane Childhood Cancer Group (version 3). 2014.

20. Scottish Intercollegiate Guidelines Network (SIGN). Long term follow up of survivors of childhood cancer. A national clinical guideline, No. 132. 2013 [cited 201628 July]; Available from: http://www.sign.ac.uk/pdf/sign76.pdf.

21. Clinical Oncology Society of Australia (COSA) Cancer Survivorship Care in Australia Position Statement Critical Components of Cancer Survivorship Care (Draft). 2015.

22. Jankovic, M., et al., Long-term survivors of childhood cancer: cure and care-the Erice Statement (2006) revised after 10 years (2016). J Cancer Surviv, 2018: p. doi:10.1007/s11764-018-0701-0.

23. Bowers, D.C., et al., Survey of long-term follow-up programs in the United States for survivors of childhood brain tumors. Pediatr Blood Cancer, 2009. 53(7): p. 1295-1301.

24. Carlson, C.A., et al., A multidisciplinary model of care for childhood cancer survivors with complex medical needs. J Pediatr Oncol Nurs, 2008. 25(1): p. 7-13.

25. Eshelman-Kent, D., et al., Cancer survivorship practices, services, and delivery: a report from the Children's Oncology Group (COG) nursing discipline, adolescentyoung adult, and late effects committees. $\mathrm{J}$ Cancer Surviv, 2011. 5(4): p. 345-57.

26. Granek, L., et al., Psychological factors impacting transition from paediatric to adult care by childhood cancer survivors. J Cancer Surviv, 2012. 6(3): p. 2609.

27. Guilcher, G.M., C. Fitzgerald, and S. Pritchard, $A$ questionnaire based review of long-term follow-up 
programs for survivors of childhood cancer in Canada. Pediatr Blood Cancer, 2009. 52(1): p. 113115.

28. Hahn, E.E. and P.A. Ganz, Survivorship Programs and Care Plans in Practice: Variations on a Theme. J Oncol Pract, 2011. 7(2): p. 70-75.

29. Kam, V., et al., Evaluation of a joint adult and pediatric clinic for cancer survivorship care. Pediatric Blood \& Cancer, 2017: p. e26476-n/a.

30. Kenney, L.B., et al., The current status of follow-up services for childhood cancer survivors, are we meeting goals and expectations: A report from the consortium for New England childhood cancer survivors. Pediatr Blood Cancer, 2011. 57(6): p. 10621066.

31. Landier, W., et al., Impact of tailored education on awareness of personal risk for therapy-related complications among childhood cancer survivors. J Clin Oncol, 2015. 33(33): p. 3887-93.

32. Langer, T., G. Henze, and J.D. Beck, Basic methods and the developing structure of a late effects surveillance system (LESS) in the long-term follow-up of pediatric cancer patients in Germany. For the German Late Effects Study Group in the German Society Pediatric Oncology and Hematology (GPOH). Med Pediatr Oncol, 2000. 34(5): p. 348-51.

33. Lindell, R.B., et al., Knowledge of diagnosis, treatment history, and risk of late effects among childhood cancer survivors and parents: The impact of a survivorship clinic. Pediatr Blood Cancer, 2015. 62(8): p. 1444-51.

34. Oeffinger, K.C., et al., Models of cancer survivorship health care: moving forward. Am Soc Clin Oncol Educ Book, 2014: p. 205-13.

35. Ristovski-Slijepcevic, S., et al., A cross-Canada survey of clinical programs for the care of survivors of cancer in childhood and adolescence. Paediatrics \& Child Health, 2009. 14(6): p. 375-378.

36. Sadak, K.T., et al., The Clinical and Research Infrastructure of a Childhood Cancer Survivor Program. J Cancer Educ, 2015. 30(3): p. 471-6.

37. Szalda, D., et al., Engagement and experience with cancer-related follow-up care among young adult survivors of childhood cancer after transfer to adult care. J Cancer Surviv, 2016. 10(2): p. 342-50.

38. Taylor, A., et al., Long-term follow-up of survivors of childhood cancer in the UK. Pediatr Blood Cancer, 2004. 42(2): p. 161-8.

39. Blaauwbroek, R., et al., Family doctor-driven followup for adult childhood cancer survivors supported by a web-based survivor care plan. J Cancer Surviv, 2012. 6(2): p. 163-171.

40. Kirchhoff, A.C., et al., Risk of hospitalization for survivors of childhood and adolescent cancer. Cancer Epidemiol Biomarkers Prev, 2014. 23(7): p. 1280-9.

41. Overholser, L.S., et al., Development of a Primary Care-Based Clinic to Support Adults With a History of Childhood Cancer: The Tactic Clinic. J Pediatr Nurs, 2015. 30(5): p. 724-31.

42. Singer, S., et al., General practitioner involvement in follow-up of childhood cancer survivors: A systematic review. Pediatr Blood Cancer, 2013. 60(10): p. 15651573.

43. Glaser, A., et al., Enhanced quality and productivity of long-term aftercare of cancer in young people. Arch Dis Child, 2013. 98(10): p. 818-24.

44. Oeffinger, K.C., P.C. Nathan, and L.C. Kremer, Challenges after curative treatment for childhood cancer and long-term follow up of survivors. Hematol Oncol Clin North Am, 2010. 24(1): p. 129-49.

45. Wallace, W.H.B., et al., Developing strategies for long term follow up of survivors of childhood cancer. BMJ, 2001. 323(7307): p. 271-274.

46. Eiser, C., et al., Follow-up after childhood cancer: Evaluation of a three-level model. Eur J Cancer, 2006. 42(18): p. 3186-3190.

47. Edgar, A.B., et al., Long-term follow-up of survivors of childhood cancer. Endocr Dev, 2009. 15: p. 159180.

48. Oeffinger, K.C. and E.S. Tonorezos, The cancer is over, now what? Cancer, 2011. 117(S10): p. 22502257.

49. Edgar, A.B., et al., Can intensity of long-term followup for survivors of childhood and teenage cancer be determined by therapy-based risk stratification? BMJ Open, 2013. 3(8).

50. Frobisher, C., et al., Risk stratification of childhood cancer survivors necessary for evidence-based clinical long-term follow-up. $\mathrm{Br} \mathrm{J}$ Cancer, 2017. 117(11): p. 1723-1731.

51. Bhatia, S. and A.T. Meadows, Long-term follow-up of childhood cancer survivors: Future directions for clinical care and research. Pediatric Blood \& Cancer, 2006. 46(2): p. 143-148.

52. Friedman, D.L., D.R. Freyer, and G.A. Levitt, Models of care for survivors of childhood cancer. Pediatr Blood Cancer, 2006. 46(2): p. 159-68.

53. Kirchhoff, A.C., et al., Childhood cancer survivors' primary care and follow-up experiences. Support Care Cancer, 2014. 22(6): p. 1629-35.

54. Nathan, P.C., et al., Family physician preferences and knowledge gaps regarding the care of adolescent and young adult survivors of childhood cancer. J Cancer Surviv, 2013.

55. Suh, E., et al., General Internists' Preferences and Knowledge About the Care of Adult Survivors of Childhood CancerA Cross-sectional Survey. Annals of Internal Medicine, 2014. 160(1): p. 11-17.

56. Cárceles-Álvarez, A., et al., Long-term follow-up of childhood cancer survivors in the Murcia Region: Preferences and attitudes of Primary Care professionals. Anales de Pediatría (English Edition), 2015. 83(4): p. 264-271.

57. Iyer, N.S., et al., Experiences with the survivorship care plan in primary care providers of childhood cancer survivors: a mixed methods approach. Supportive Care in Cancer, 2017: p. 1-9.

58. Blaauwbroek, R., et al., The willingness of general practitioners to be involved in the follow-up of adult survivors of childhood cancer. J Cancer Surviv, 2007. 1(4): p. 292-297.

59. Aziz, N.M., et al., Comprehensive long-term follow-up programs for pediatric cancer survivors. Cancer, 2006. 107(4): p. 841-8.

60. Berg, C., et al., Providers' Perspectives of Survivorship Care for Young Adult Survivors of Childhood Cancer. J Cancer Educ, 2016. 31(1): p. 3138.

61. Bober, S.L., et al., Caring for cancer survivors: $a$ survey of primary care physicians. Cancer, 2009. 115(18 Suppl): p. 4409-18.

62. Blaauwbroek, R., et al., Shared care by paediatric oncologists and family doctors for long-term followup of adult childhood cancer survivors: a pilot study. Lancet Oncol, 2008. 9(3): p. 232-8.

63. Szalda, D., et al., Internet-Based Survivorship Care Plans for Adult Survivors of Childhood Cancer: A 
Pilot Study. J Adolesc Young Adult Oncol, 2016. 5(4): p. 351-354.

64. Klosky, J.L., et al., Factors influencing long-term follow-up clinic attendance among survivors of childhood cancer. J Cancer Surviv, 2008.

65. Sima, J.L., S.M. Perkins, and D.A. Haggstrom, Primary care physician perceptions of adult survivors of childhood cancer. J Pediatr Hematol Oncol, 2014. 36(2): p. 118-24.

66. Zebrack, B., et al., Assessing the health care needs of adolescent and young adult cancer patients and survivors. Cancer, 2006. 107(12): p. 2915-23.

67. Park, E.R., et al., Childhood Cancer Survivor Study participants' perceptions and knowledge of health insurance coverage: implications for the Affordable Care Act. J Cancer Surviv, 2012. 6(3): p. 251-9.

68. Berg, C.J., et al., Young Adult Cancer Survivors' Experience with Cancer Treatment and Follow-Up Care and Perceptions of Barriers to Engaging in Recommended Care. J Cancer Educ, 2016. 31(3): p. 430-442.

69. Michel, G., et al., Physicians' experience with followup care of childhood cancer survivors - challenges and needs. Swiss Med Wkly, 2017. 147: p. w14457.

70. Ishida, Y., et al., Physician preferences and knowledge regarding the care of childhood cancer survivors in Japan: a mailed survey of the Japanese society of pediatric oncology. Jpn J Clin Oncol, 2012. 42(6): p. 513-21.

71. Henderson, T.O., et al., Physician Preferences and Knowledge Gaps Regarding the Care of Childhood Cancer Survivors: A Mailed Survey of Pediatric Oncologists. J Clin Oncol, 2010. 28(5): p. 872-877.

72. Vetsch, J., et al., Parents' preferences for the organisation of long-term follow-up of childhood cancer survivors. European Journal of Cancer Care, 2018. 27(2): p. e12649.

73. Michel, G., et al., Follow-up care after childhood cancer: Survivors' expectations and preferences for care. Eur J Cancer, 2009. 45(9): p. 1616-1623

74. Michel, G., et al., Preferences for long-term follow-up care in childhood cancer survivors. Eur J Cancer Care (Engl), 2016. 25(6): p. 1024-1033.

75. Christen, S., et al., Preferences for the organization of long-term follow-up in adolescent and young adult cancer survivors. Support Care Cancer, 2016. 24(8): p. 3425-36.

76. Absolom, K., et al., Predictors of clinic satisfaction among adult survivors of childhood cancer. Eur $\mathrm{J}$ Cancer, 2006. 42(10): p. 1421-7.

77. van Laar, M., et al., The impact of a managed transition of care upon psychosocial characteristics and patient satisfaction in a cohort of adult survivors of childhood cancer. Psychooncology, 2013.

78. Warnock, C., et al., Emerging nursing roles for late effects care for children and young adults with cancer. Eur J Oncol Nurs, 2013. 17(2): p. 242-9.

79. D'Agostino, N.M. and K. Edelstein, Psychosocial challenges and resource needs of young adult cancer survivors: implications for program development. $\mathrm{J}$ Psychosoc Oncol, 2013. 31(6): p. 585-600.

80. Lie, H.C., et al., Providing information about late effects after childhood cancer: Lymphoma survivors' preferences for what, how and when. Patient Education and Counseling, 2015. 98(5): p. 604-611.

81. Mayes, J., et al., Health promotion and information provision during long-term follow-up for childhood cancer survivors: A service evaluation. Pediatr Hematol Oncol, 2016: p. 1-12.
82. Zebrack, B., Information and service needs for young adult cancer survivors. Support Care Cancer, 2009. 17(4): p. 349-57.

83. Galan, S., et al., What are the needs of adolescents and young adults after a cancer treatment? A Delphi study. Eur J Cancer Care (Engl), 2017. 26(2): p. e12488

84. Aukema, E.J., et al., Explorative study on the aftercare of pediatric brain tumor survivors: a parents' perspective. Support Care Cancer, 2011. 19(10): p. 1637-46.

85. Meacham, L.R., et al., Primary care providers as partners in long-term follow-up of pediatric cancer survivors. J Cancer Surviv, 2012. 6(3): p. 270-7.

86. Dilley, K.J. and B. Lockart, The pediatric brain tumor late effects clinic. Cancer Treat Res, 2009. 150: p. 97109.

87. Mertens, A.C., et al., Improving health care for adult survivors of childhood cancer: recommendations from a delphi panel of health policy experts. Health Policy, 2004. 69(2): p. 169-78.

88. Earle, E.A., et al., Follow-up care for childhood cancer survivors: a focus group analysis. Eur J Cancer, 2005. 41(18): p. 2882-6.

89. Sutradhar, R., et al., Specialized survivor clinic attendance is associated with decreased rates of emergency department visits in adult survivors of childhood cancer. Cancer, 2015.

90. Firth, E.R., N. Davies, and R. Skinner, Views of childhood cancer survivors and their families on the provision and format of a treatment summary. J Pediatr Hematol Oncol, 2013. 35(3): p. 193-6.

91. Spain, P.D., et al., Response to a treatment summary and care plan among adult survivors of pediatric and young adult cancer. J Oncol Pract, 2012. 8(3): p. 196202.

92. Warner, E.L., et al., Health care concerns of rural childhood cancer survivors. J Health Care Poor Underserved, 2014. 25(2): p. 901-12.

93. Shalom, M.M., et al., Do survivorship care plans make a difference? A primary care provider perspective. $\mathrm{J}$ Oncol Pract, 2011. 7(5): p. 314-8.

94. Oeffinger, K.C., et al., Increasing rates of breast cancer and cardiac surveillance among high-risk survivors of childhood Hodgkin lymphoma following a mailed, one-page survivorship care plan. Pediatr Blood Cancer, 2011. 56(5): p. 818-24.

95. Poplack, D.G., et al., Childhood cancer survivor care: development of the Passport for Care. Nat Rev Clin Oncol, 2014. 11(12): p. 740-750.

96. Casillas, J., et al., How confident are young adult cancer survivors in managing their survivorship care? A report from the LIVESTRONG Survivorship Center of Excellence Network. J Cancer Surviv, 2011. 5(4): p. 371-381.

97. Sadak, K.T., A. DiNofia, and G. Reaman, PatientPerceived Facilitators in the Transition of Care for Young Adult Survivors of Childhood Cancer. Pediatric Blood \& Cancer, 2013. 60(8): p. 1365-1368.

98. Oeffinger, K.C., et al., Grading of late effects in young adult survivors of childhood cancer followed in an ambulatory adult setting. Cancer, 2000. 88(7): p. 1687-95.

99. Jefford, M., et al., Implementing improved posttreatment care for cancer survivors in England, with reflections from Australia, Canada and the USA. Br J Cancer, 2013. 108(1): p. 14-20.

100. Signorelli, C., et al., The impact of long-term follow-up care for childhood cancer survivors: A 
systematic review. Crit Rev Oncol Hematol, 2017. 114: p. 131-138

101. Jacobsen, P.B., et al., Systematic Review of the Impact of Cancer Survivorship Care Plans on Health Outcomes and Health Care Delivery. Journal of Clinical Oncology, 2018: p. doi: JCO.2018.77.7482.
102. Hewitt, M., S. Greenfield, and E. Stovall, eds. From cancer patient to cancer survivor: Lost in transition. 2006, The National Academies Press: Washington DC.

103. Blaauwbroek, R., et al., Late effects in adult survivors of childhood cancer: the need for life-long follow-up. Ann Oncol, 2007. 18(11): p. 1898-1902. 
Table 1: Search strategy

The Dutch Childhood Cancer Group (DCOG) has performed a PubMed literature search for papers published between 1999 and 2009 for questions 1, 2 and 3. This search was updated for the current guideline until 8 February 2017.

\begin{tabular}{|c|c|}
\hline Search 1: & $\begin{array}{l}\text { infant OR infan* OR newborn OR newborn* OR new-born* OR baby OR baby* OR } \\
\text { babies OR neonat* OR perinat* OR postnat* OR child OR child* OR schoolchild* OR } \\
\text { schoolchild OR school child OR school child* OR kid OR kids OR toddler* OR } \\
\text { adolescent OR adoles* OR teen* OR boy* OR girl* OR minors OR minors* OR } \\
\text { underag* OR under ag* OR juvenil* OR youth* OR kindergar* OR puberty OR puber* } \\
\text { OR pubescen* OR prepubescen* OR prepuberty* OR paediatrics OR paediatric* OR } \\
\text { paediatric* OR peadiatric* OR schools OR nursery school* OR preschool* OR pre } \\
\text { school* OR primary school* OR secondary school* OR elementary school* OR } \\
\text { elementary school OR high school* OR highschool* OR school age OR schoolage OR } \\
\text { school age* OR schoolage* OR infancy OR schools, nursery OR infant, newborn }\end{array}$ \\
\hline Search 2: & $\begin{array}{l}\text { (((leukemia OR leukemi* OR leukaemi* OR (childhood ALL) OR AML OR lymphoma } \\
\text { OR lymphom* OR hodgkin OR hodgkin* OR T-cell OR B-cell OR non-hodgkin OR } \\
\text { sarcoma OR sarcom* OR sarcoma, Ewing's OR Ewing* OR osteosarcoma OR } \\
\text { osteosarcom* OR wilms tumor OR wilms* OR nephroblastom* OR neuroblastoma OR } \\
\text { neuroblastom* OR rhabdomyosarcoma OR rhabdomyosarcom* OR teratoma OR } \\
\text { teratom* OR hepatoma OR hepatom* OR hepatoblastoma OR hepatoblastom* OR PNET } \\
\text { OR medulloblastoma OR medulloblastom* OR PNET* OR neuroectodermal tumors, } \\
\text { primitive OR retinoblastoma OR retinoblastom* OR meningioma OR meningiom* OR } \\
\text { glioma OR gliom*) OR (paediatric oncology OR paediatric oncology)) OR (childhood } \\
\text { cancer OR childhood tumor OR childhood tumors)) OR (brain tumor* OR brain tumour* } \\
\text { OR brain neoplasms OR central nervous system neoplasm OR central nervous system } \\
\text { neoplasms OR central nervous system tumor* OR central nervous system tumour* OR } \\
\text { brain cancer* OR brain neoplasm* OR intracranial neoplasm*) OR (leukemia } \\
\text { lymphocytic acute) OR (leukemia, lymphocytic, acute[mh]) OR cancer OR cancers OR } \\
\text { cancer* OR oncology OR oncolog* OR neoplasm OR neoplasms OR neoplasm* OR } \\
\text { carcinoma OR carcinom* OR tumor OR tumour OR tumor* OR tumour* OR tumors OR } \\
\text { tumours OR malignan* OR malignant OR hematooncological OR hemato oncological } \\
\text { OR hemato-oncological OR hematologic neoplasms OR hematolo* }\end{array}$ \\
\hline Search 3: & $\begin{array}{l}\text { Survivor OR survivors OR Long-Term Survivors OR Long Term Survivors OR Long- } \\
\text { Term Survivor OR Survivor, Long-Term OR Survivors, Long-Term OR survivo* OR } \\
\text { survivi* }\end{array}$ \\
\hline Search 4: & $\begin{array}{l}\text { continuity of patient care OR quality assurance, health care OR aftercare OR delivery of } \\
\text { health care OR home care OR models of care OR shared care OR health care OR delivery } \\
\text { of care OR follow-up care OR models, organisational OR models, organizational OR } \\
\text { referral and consultation OR health services need and demand OR attitude of health } \\
\text { personnel OR long-term care OR transition of care OR "transfer of care" OR transitional } \\
\text { model OR transition-based model } \\
\text { OR } \\
\text { nurse[tiab] OR nursing[sh] OR nursing[tiab] OR nurse* OR nurses OR general } \\
\text { practitioner OR family practice OR oncologist[All Fields] OR oncologists OR } \\
\text { oncologist* OR health personnel OR "health care provider" OR "primary care provider" } \\
\text { OR "secondary care provider" OR "care provider" OR physicians, family OR primary } \\
\text { care physician OR family physician OR "health care professional" }\end{array}$ \\
\hline \multicolumn{2}{|c|}{ Search 1 AND 2 AND 3 AND 4} \\
\hline
\end{tabular}


Table 2: Conclusions of evidence from the systematic literature search for models of LTFU care for childhood, adolescent and young adult cancer survivors

\section{Organisation of LTFU}

Theoretical LTFU care models and LTFU care models used in practice

Risk-based models for LTFU care:

- $\quad$ Cancer centre-delivered care in the paediatric oncology clinic, medical oncology clinic or LTFU clinic

- $\quad$ Shared care between treatment hospital and local hospital or primary care

- $\quad$ Primary care physician-led LTFU care (community-based care)

$[16,23-39,41-45,51-53]$

- $\quad$ Specialist nurse-led supported LTFU care

- Self-management supported by health professionals within a shared care or primary care model

- $\quad$ Certain groups might need specialised clinics such as survivors with HSCT and CNS tumour survivors

$[43-45,51,52]$

LTFU care is provided in a multidisciplinary setting [16, 24-30, 32, 34-36, 38, 41, 42, 53]

Risk stratification is an important component of LTFU care to assign the level and model of

LTFU care [43-45]

Opinions of survivors and healthcare providers about models of LTFU care

Primary care physicians are willing to care for childhood cancer survivors in collaboration

with a LTFU clinic / cancer centre within a shared care model $[42,54-56,61,62,69,85,93]$

Primary care physicians may feel uncomfortable in caring for adult survivors of childhood cancer [54-57]

Paediatric oncologists preferably provide care in a multidisciplinary team $[16,70,71]$

Paediatric oncologists feel comfortable in caring for younger childhood cancer survivors, but

less comfortable in caring for older childhood cancer survivors [70, 71]

Survivors are satisfied with a shared-care model [37,62]

Survivors want to be able to make their own choice of care provider [67]

Survivors rate consultant-led follow-up higher than general practitioner-led follow-up, nurse-

led follow-up and postal/telephone follow-up [73-75]

Survivors see the paediatric/adolescent clinic as appropriate location for their LTFU, and do not see a need to change [81]

Parents prefer the paediatric oncologist model and multidisciplinary team model above the medical oncologist model and general practitioner model [72]

Level C

Expert opinion

Level C

Level C

Level C

Level C

Level C

Level C

Level C

Level C

Level C

Level C

Level C

Opinions of survivors and healthcare providers about barriers to LTFU care

- Lack of a LTFU programme [16, 80, 87]

- Lack of communication between primary care physician and paediatric oncologists [42, $58,59,68]$

- Confusion about role of survivorship programs, oncologists and primary care provider $[16,25,68]$

- Lack of support and staff to provide LTFU care [16, 59, 66, 68]

Level C

- Limited access to refer survivors to specialist care [61]

- Lack of adequate insurance or funding for LTFU care [16, 23, 25, 59, 65-68]

- Lack of time/ high workload [16, 25, 42, 58, 59, 61, 65, 68, 69]

- Distance to clinic for survivors $[16,68]$

Inability to locate adult survivors $[16,23]$

Opinions of survivors and healthcare providers about facilitators to LTFU care

- Access to LTFU care, including access to cancer survivor specialists, access to support services, like social work and psychology, ability to telephone or email specialist for advice, and more medical/support staff in primary care office $[53-55,58,60,61,69,85$, 90, 91, 93]

- Adequate insurance [66]

Effectiveness and feasibility of LTFU care models in practice

Shared-care for childhood cancer survivors by the family doctor and paediatric oncologist is feasible [62]

Childhood cancer survivors are satisfied with LTFU care at a paediatric or adult LTFU clinic or shared-care between primary care provider and LTFU clinic [37, 62, 76, 77]
Level C

Level C 


\begin{tabular}{|c|c|}
\hline $\begin{array}{l}\text { There were no significant differences in engagement in LTFU care between primary care } \\
\text { providers and subspecialty survivorship care providers [37] }\end{array}$ & Level C \\
\hline LTFU care for childhood cancer survivors in a paediatric LTFU clinic is feasible [64] & Level C \\
\hline $\begin{array}{l}\text { LTFU care for childhood cancer survivors by a multidisciplinary transition programme in an } \\
\text { adult setting is feasible [98] }\end{array}$ & Level C \\
\hline $\begin{array}{l}\text { LTFU care for childhood cancer survivors by a joint adult/paediatric LTFU care at a } \\
\text { paediatric hospital is feasible [29] }\end{array}$ & Level C \\
\hline \multicolumn{2}{|l|}{ Effectiveness and feasibility risk stratification } \\
\hline $\begin{array}{l}\text { It is feasible to identify and appropriately categorize childhood cancer survivors by the } \\
\text { severity (grade) of the expected late effects }[34,43,44,46-50]\end{array}$ & Level C \\
\hline \multicolumn{2}{|l|}{ Personnel involved in LTFU care } \\
\hline \multicolumn{2}{|l|}{ Theoretical LTFU care models and LTFU care models used in practice } \\
\hline $\begin{array}{l}\text { LTFU care is provided in a multidisciplinary setting, including a paediatric oncology } \\
\text { physician, (paediatric oncology) nurse practitioner, primary care physicians (paediatrician, } \\
\text { internist, general practitioner), radiation oncologist, medical oncologist, (neuro)psychologist, } \\
\text { (paediatric) social worker, dietician, clinical research associate, physician assistants, clinical } \\
\text { pharmacist, physiotherapist, (paediatric) endocrinologist, non-clinical staff (program } \\
\text { manager, clinical research associate, database manager, administrative support) [16, 24-30, } \\
32,34-36,38,41,42,53]\end{array}$ & Level C \\
\hline LTFU care should be coordinated by a key worker $[43,44,86]$ & Expert opinion \\
\hline \multicolumn{2}{|c|}{ Opinions of survivors and healthcare providers about who should be involved LTFU care } \\
\hline Childhood cancer survivors want to be able to make their own choice of care provider [67] & Level C \\
\hline $\begin{array}{l}\text { Childhood cancer survivors prefer hospital-based LTFU, while others would rather attend } \\
\text { LTFU at their primary care provider [72-75] }\end{array}$ & Level C \\
\hline Nurses can have important roles in the care for childhood cancer survivors [78] & Level C \\
\hline $\begin{array}{l}\text { Paediatric oncologists feel comfortable in caring for younger childhood cancer survivors, but } \\
\text { less comfortable in caring for older childhood cancer survivors }[70,71]\end{array}$ & Level C \\
\hline \multicolumn{2}{|l|}{ Opinions of survivors and healthcare providers about barriers to LTFU care } \\
\hline Lack of staff to provide LTFU care $[16,59]$ & Level C \\
\hline \multicolumn{2}{|l|}{ Effectiveness and feasibility of LTFU care models in practice } \\
\hline $\begin{array}{l}\text { Childhood cancer survivors who attend a paediatric clinic are significantly more [76] or } \\
\text { equally [77] satisfied with LTFU care than survivors attending an adult clinic }\end{array}$ & Level C \\
\hline \multicolumn{2}{|l|}{ Components of LTFU care } \\
\hline \multicolumn{2}{|l|}{ Theoretical LTFU care models and LTFU care models used in practice } \\
\hline $\begin{array}{l}\text { Important components of LTFU care include a survivorship care plan, patient/survivor and } \\
\text { parent education, and planned transition to assign the level and model of LTFU care [16, 25- } \\
30,33-36,39,41-45,86]\end{array}$ & Level C \\
\hline $\begin{array}{l}\text { Training, development and education programmes for professionals caring for survivors are } \\
\text { important }[30,34,42,43]\end{array}$ & Level C \\
\hline $\begin{array}{l}\text { Audits to test and review if the clinical level and model of care is still appropriate are } \\
\text { important [43] }\end{array}$ & Expert opinion \\
\hline \multicolumn{2}{|l|}{ Opinions of survivors and healthcare providers about requirements of LTFU care } \\
\hline $\begin{array}{l}\text { Survivors and parents have wide ranging requirements for follow-up care, including } \\
\text { information and counselling about late effects and preventive care, psychosocial support, } \\
\text { parenting issues, and education for primary care providers and parents }[66,67,72-75,79-84]\end{array}$ & Level C \\
\hline Survivors are more satisfied with LTFU care when discussing topics they want [73] & Level C \\
\hline Survivors are willing to transition to adult survivorship care $[37,97]$ & Level C \\
\hline \multicolumn{2}{|l|}{ Opinions of survivors and healthcare providers about barriers to LTFU care } \\
\hline $\begin{array}{ll}- & \text { Lack of a LTFU programme }[16,80,87] \\
\text { - } & \text { Lack of knowledge or awareness about late effects, survivorship issues and needs }[10,16, \\
& 23,25,37,42,54,55,57-59,61,62,65,66,69,70,73,87,92] \\
- & \text { Lack of knowledge about late effects among survivors and parents }[10,79,84,88] \\
\text { - } & \text { Survivor-related psychosocial barriers (fear, avoidance) }[68,96] \\
- & \text { Lack of knowledge and familiarity of LTFU guidelines }[54,55,61,65,66,70] \\
- & \text { Low confidence in managing their survivorship care among survivors }[96]) \\
- & \text { Difficulties organising an appointment (time, distance, scheduling) }[68] \text { or finding the } \\
& \text { right place to go [80] } \\
\text { - } & \text { Lack of a transition program from paediatric to adult healthcare }[87] \\
\text { - } & \text { Inadequate access to survivors' cancer treatment history }[61,65]\end{array}$ & Level C \\
\hline
\end{tabular}




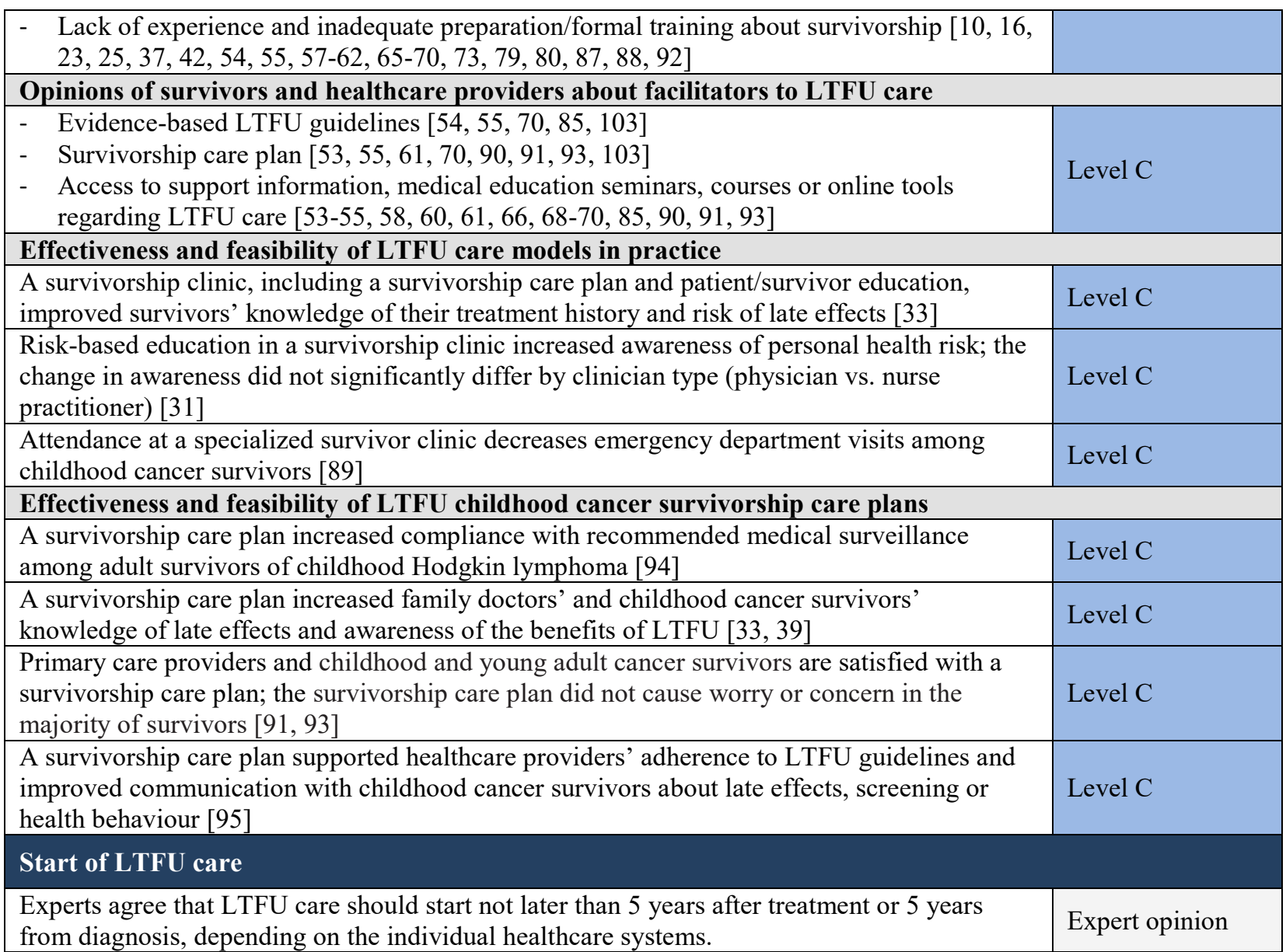

LTFU, Long-term follow-up 
Table 3: Recommendations for the LTFU care of childhood, adolescent and young adult cancer survivors based on low quality evidence and expert opinion.

\begin{tabular}{l} 
General recommendation \\
\hline $\begin{array}{l}\text { We recommend that LTFU care should be available and accessible for all childhood, adolescent } \\
\text { and young adult cancer survivors throughout their lifespan. }\end{array}$ \\
Organisation of LTFU care \\
\hline $\begin{array}{l}\text { We recommend that LTFU care for survivors of childhood, adolescent and young adult cancer } \\
\text { should: } \\
\text { be provided in or under the guidance of a cancer survivorship expert service or cancer } \\
\text { centre } \\
\text { provide multidisciplinary care }\end{array}$
\end{tabular}

We recommend that the survivor and healthcare provider make a joint decision for the optimal model of LTFU care, ${ }^{1}$ based on previous cancer treatment, health conditions, survivor preferences and the healthcare system.

To provide LTFU care for survivors of childhood, adolescent and young adult cancer we recommend:

$\circ$ To have commitment of the (national and local) healthcare providers (systems) and insurers

To have sufficient time for consultation

\section{Personnel involved in LTFU care}

We recommend that each survivor can make their own informed choice for a healthcare provider after informed discussion with the survivorship team.

We recommend that the cancer survivorship expert centre that will organize LTFU care includes:

○ Key worker/coordinator

- Lead doctor specialised in late effects

- Nurse practitioner

Multidisciplinary expert team of specialists ${ }^{2}$

The possibility of consulting specific specialist ${ }^{3}$

\section{Components of LTFU care}

We recommend that LTFU care for survivors of childhood, adolescent and young adult cancer include:

- Surveillance and preventive strategies based on published evidence based guidelines

- Coordination of care (particularly in shared care models)

Education for professionals

Education of survivors, families \& carers

$\circ$ Coordination of scientific research

We recommend that the cancer survivorship expert centre provides:

$\circ$ An individualized survivorship care plan

- including a treatment summary with risk stratification care plan

- Patient/survivor and parent education to support effective self-management

- A plan for transition of care:

- from active treatment to LTFU

- from survivorship expert centre to primary care (for low risk survivors)

- from paediatric to adult health services

\section{Start of LTFU care}

We recommend that LTFU care should start not later than $\mathbf{5}$ years after treatment or $\mathbf{5}$ years from diagnosis, depending on the individual healthcare systems. 
LTFU, Long-term follow-up care

${ }^{1}$ Self-management with primary care support for adult survivors; follow-up at primary care level or by a nurse experienced in management of late effects, followed by supported self-management; follow-up at cancer survivorship expert centre; or shared care between survivorship expert centre and primary care or paediatric centres.

${ }^{2}$ paediatric oncologist/haematologist, (neuro-) psychologist, cardiologist, endocrinologist, medical oncologist, haematologist, rehabilitation physician, occupational worker, radiotherapist, social worker

${ }^{3}$ pulmonologist, nephrologist, neurologist, neurosurgeon, ear nose and throat specialist, ophthalmologist, gynaecologist, dermatologist, insurance worker, urologists, general internal medicine 


\section{Figure 1: Flowchart of literature search}

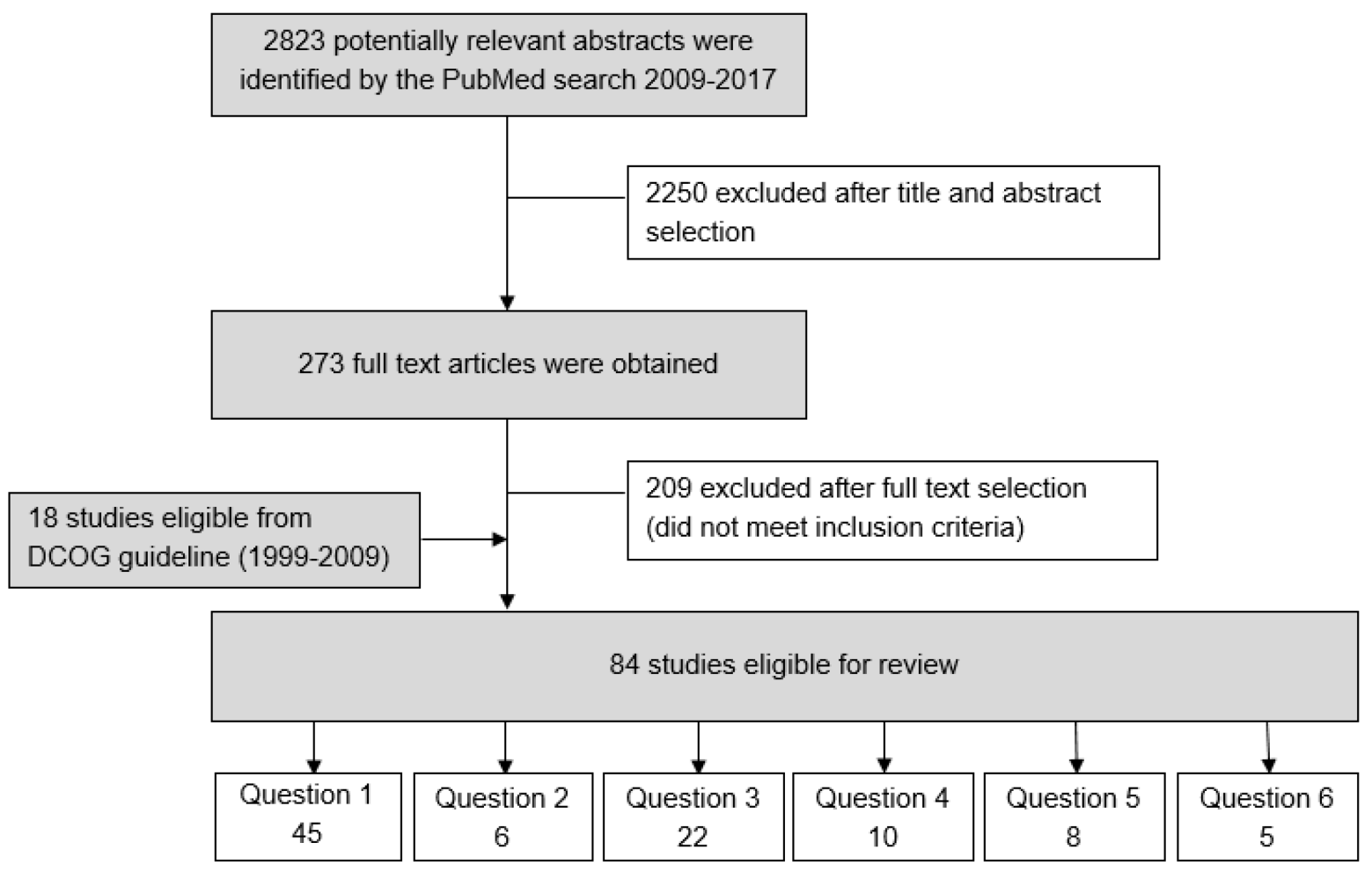


Online supplemental information

Online Resource 1: Assessment of concordance and discordance between existing guidelines for childhood and adolescent cancer survivors

Online Resource 2: Grading the Levels of Evidence and Strength of Recommendations

Online Resource 3: Conclusions of evidence tables models of care

Online Resource 4: Evidence Tables (separate file) 